\title{
Preliminary investigation on Salmonella and Escherichia coli contamination of chicken meat sold at small-scale farm shops in Kandy District, Sri Lanka
}

\author{
BYI Gamage ${ }^{1}$, DS Thilakarathne ${ }^{2}$, KGR Abayawansha ${ }^{1}$, RS Kalupahana ${ }^{1}$
}

Introduction and Objectives:Salmonella and Escherichia coli are two important foodborne pathogens that frequently contaminate animal origin products, particularly chicken meat, the most consumed meat variety in Sri Lanka (SL). Though many Sri Lankans purchase frozen branded chicken meat from supermarkets and other sales outlets, a certain fraction of the population prefers fresh/thawed chicken meat sold at small-scale farm shops. Compared to branded chicken produced by integrated poultry business, small scale chicken production and distribution network appears to be less regulated. A study conducted during 2010-2011 has shown chicken meat sold at small-scale farm shops in Kandy district, SL is highly contaminated with antimicrobial-resistant E. coli and Salmonella. The present study aims to compare the level of contamination of chicken meat sold at small-scale farm shops with Salmonella and E. coli, in the same study area a decade later.

Methods: The chicken meat was purchased from 37 farm shops representing the 9 most populous of the 20 divisional secretariat divisions in Kandy district. Isolation and identification of Salmonella and E. coli were performed according to ISO 6579 and modified SLS 516: part 3: 1982 protocols respectively.

Results:Salmonella contamination was detected in 22 / 37 (59.5\%) meat samples whereas 26 / 37 (70.3\%) meat samples were contaminated with E. coli. When compared with the study conducted a decade ago, the level of Salmonella contamination has increased from $47.2 \%$ to $59.5 \%$ while $E$. coli detection has decreased from $100 \%$ to $70.3 \%$.

Conclusions: The current study shows consumers are at high risk of getting exposed to foodborne pathogens through meat sold at small-scale farm shops. Awareness programs and legislation implemented in the last decade have been inadequate to reduce contamination to a significant level. The contamination could be due to substandard operational hygiene. It is timely to regulate these small-scale farm shops via a central authority to secure food safety.

Keywords: Farm-shops, chicken, meat, foodborne, E. coli, Salmonella

\footnotetext{
${ }^{1}$ Department of Veterinary Public Health and Pharmacology, Faculty of Veterinary Medicine and Animal Science, University of Peradeniya, Sri Lanka

${ }^{2}$ Department of Pathobiology, Faculty of Veterinary Medicine and Animal Science, University of Peradeniya, Sri Lanka
} 